\title{
Geology of the Selk crater region on Titan from Cassini VIMS observations
}

\author{
Jason M. Soderblom ${ }^{\mathrm{a}, *}$, Robert H. Brown ${ }^{\mathrm{a}}$, Laurence A. Soderblom ${ }^{\mathrm{b}}$, Jason W. Barnes ${ }^{\mathrm{c}}$, Ralf Jaumann ${ }^{\mathrm{d}}$, \\ Stéphane Le Mouélic ${ }^{e}$, Christophe Sotin ${ }^{f, e}$, Katrin Stephan ${ }^{d}$, Kevin H. Baines ${ }^{f}$, Bonnie J. Buratti ${ }^{f}$, \\ Roger N. Clark ${ }^{\mathrm{g}}$, Philip D. Nicholson ${ }^{\mathrm{h}}$
}

\author{
${ }^{a}$ Lunar and Planetary Laboratory, University of Arizona, Tucson, AZ 85721, USA \\ ${ }^{\mathrm{b}}$ US Geological Survey, Flagstaff, AZ 86001, USA \\ ${ }^{\mathrm{c}}$ Department of Physics, University of Idaho, Moscow, ID 83844, USA \\ ${ }^{\mathrm{d}} \mathrm{DLR}$, Institute of Planetary Research, Berlin, Germany \\ e Université de Nantes, Laboratoire de Planétologie et Géodynamique, Nantes Cedex 03, France \\ ${ }^{\mathrm{f}}$ Jet Propulsion Laboratory, California Institute of Technology, Pasadena, CA 91109, USA \\ ${ }^{g}$ US Geological Survey, Denver, CO 80225, USA \\ h Department of Astronomy, Cornell University, Ithaca, NY 14853, USA
}

\section{A R T I C L E I N F O}

\section{Article history:}

Received 25 July 2009

Revised 22 February 2010

Accepted 4 March 2010

Available online 11 March 2010

\section{Keywords:}

Titan

Saturn, Satellites

Satellites, Surfaces

Impact processes

Geological processes

\begin{abstract}
A B S T R A C T
Observations of Titan obtained by the Cassini Visual and Infrared Mapping Spectrometer (VIMS) have revealed Selk crater, a geologically young, bright-rimmed, impact crater located $\sim 800 \mathrm{~km}$ northnorthwest of the Huygens landing site. The crater rim-crest diameter is $\sim 90 \mathrm{~km}$; its floor diameter is $\sim 60 \mathrm{~km}$. A central pit/peak, 20-30 km in diameter, is seen; the ratio of the size of this feature to the crater diameter is consistent with similarly sized craters on Ganymede and Callisto, all of which are dome craters. The VIMS data, unfortunately, are not of sufficient resolution to detect such a dome. The inner rim of Selk crater is fluted, probably by eolian erosion, while the outer flank and presumed ejecta blanket appear dissected by drainages (particularly to the east), likely the result of fluvial erosion. Terracing is observed on the northern and western walls of Selk crater within a 10-15 km wide terrace zone identified in VIMS data; the terrace zone is bright in SAR data, consistent with it being a rough surface. The terrace zone is slightly wider than those observed on Ganymede and Callisto and may reflect differences in thermal structure and/or composition of the lithosphere. The polygonal appearance of the crater likely results from two preexisting planes of weakness (oriented at azimuths of $21^{\circ}$ and $122^{\circ}$ east of north). A unit of generally bright terrain that exhibits similar infrared-color variation and contrast to Selk crater extends east-southeast from the crater several hundred kilometers. We informally refer to this terrain as the Selk "bench." Both Selk and the bench are surrounded by the infrared-dark Belet dune field. Hypotheses for the genesis of the optically bright terrain of the bench include: wind shadowing in the lee of Selk crater preventing the encroachment of dunes, impact-induced cryovolcanism, flow of a fluidized-ejecta blanket (similar to the bright crater outflows observed on Venus), and erosion of a streamlined upland formed in the lee of Selk crater by fluid flow. Vestigial circular outlines in this feature just east of Selk's ejecta blanket suggest that this might be a remnant of an ancient, cratered crust. Evidently the southern margin of the feature has sufficient relief to prevent the encroachment of dunes from the Belet dune field. We conclude that this feature either represents a relatively high-viscosity, fluidizedejecta flow (a class intermediate to ejecta blankets and long venusian-style ejecta flows) or a streamlined upland remnant that formed downstream from the crater by erosive fluid flow from the west-northwest. (c) 2010 Elsevier Inc. All rights reserved.
\end{abstract}

\section{Introduction}

Only a small number of impact craters on Titan's surface have been identified from observations by the Cassini Visual and Infrared Mapping Spectrometer (VIMS; Brown et al., 2004), Imaging Science Subsystem (ISS; Porco et al., 2004), and Synthetic Aperture

\footnotetext{
* Corresponding author. Fax: +16076219692.

E-mail address: jasons@lpl.arizona.edu (J.M. Soderblom).
}

RADAR (SAR; Elachi et al., 2004) instruments (e.g., Lorenz et al., 2007; Jaumann and Neukum, 2009). All of these are larger than $5 \mathrm{~km}$ (Jaumann and Neukum, 2009), consistent with models that predict smaller bolides are unlikely to survive passage through Titan's atmosphere (e.g., Korycansky and Zahnle, 2005). These include Sinlap crater, investigated by Elachi et al. (2006), Stofan et al. (2006), Soderblom et al. (2007b), and Le Mouélic et al. (2008), Menrva crater, discussed by Elachi et al. (2006) and Stofan et al. (2006), Ksa crater, described in Lorenz et al. (2007), Afekan 
crater, located $25.8^{\circ} \mathrm{N}, 200.3^{\circ} \mathrm{W}$ (USGS/IAU Gazetteer of Planetary Nomenclature, http://www.planetarynames.wr.usgs.gov), and a few unnamed craters (cf. Porco et al., 2005; Lorenz et al., 2007; Jaumann and Neukum, 2009; Wood et al., 2010). An additional $\sim 40$ candidate impact craters have been identified in Cassini observations of Titan (Jaumann and Neukum, 2009; Wood et al., 2010). From the observed crater population, Lorenz et al. (2007) concluded Titan's average surface is geologically young: $\sim 100 \mathrm{Ma}$ to $1 \mathrm{Ga}$.

The large impact structures thus far seen on Titan exhibit varying degrees of degradation by fluvial and eolian erosion and possibly, viscous relaxation and burial by organics (Elachi et al., 2006; Stofan et al., 2006; Soderblom et al., 2007b; Lorenz et al., 2007; Le Mouélic et al., 2008), consistent with a broad range of ages. Several larger craters reportedly exhibit unique morphologies, lacking the central peaks or pits (Elachi et al., 2006; Stofan et al., 2006; Wood et al., 2010) expected of complex craters on icy satellites (e.g., Passey and Shoemaker, 1982; Schenk, 1991, 1993).

The transition from simple to complex craters results from slumping of the crater walls when some strength threshold of the crater wall is exceeded by the stress induced by gravitational force (Melosh and Ivanov, 1999). The crater diameter at which this transition occurs is inversely proportional to gravitational acceleration (Pike, 1980). Target material strength, however, does play a role as evidenced by the significant offset of this transition diameter to smaller diameters for the icy satellites (Schenk, 1991; Schenk et al., 2004). On Titan, the transition from simple to complex craters should occur at a crater diameter of $\sim 2-3 \mathrm{~km}$ (cf. Schenk, 2002, Fig. 2). Korycansky and Zahnle (2005) predict the smallest craters that should be expected on Titan are $\sim 2 \mathrm{~km}$ in diameter; it is therefore likely that few or no simple craters exist on Titan.

Selk, a bright-rimmed crater $\sim 90 \mathrm{~km}$ in diameter, located at $7.0^{\circ} \mathrm{N}, 199.0^{\circ} \mathrm{W}, \sim 800 \mathrm{~km}$ north-northwest of the Huygens landing site (cf. Fig. 1), was best observed in data acquired by Cassini VIMS during the 35th, 38th, and 40th flybys of Titan (T35, T38, and T40). The size of this impact structure places it well within the complex crater regime for icy satellites (cf. Schenk, 2002). Wood et al.

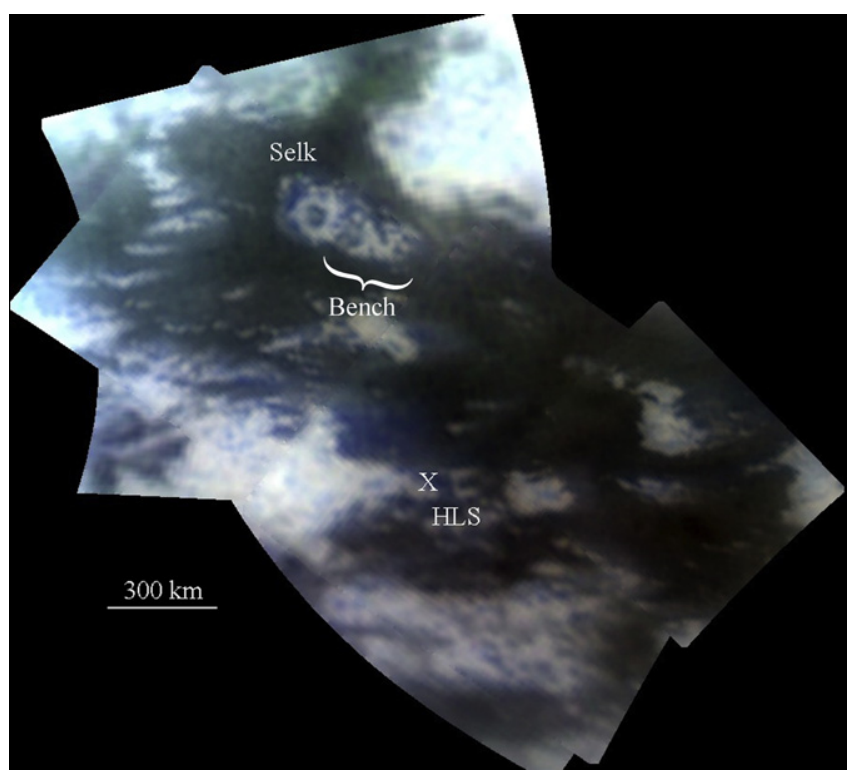

Fig. 1. VIMS color mosaic $(\mathrm{RGB}=2.0,1.6,1.3 \mu \mathrm{m})$ of the Selk crater region. The "bench," the generally optically bright unit associated with Selk crater, extends east-southeast from Selk crater several hundred kilometers. The Huygens landing site (HLS) is located southeast of Selk crater (indicated with an X in the figure). Infrared-dark material, inferred to be dunes, is seen to surround Selk crater and the bench.
(2009) concluded from Cassini SAR observations that Selk crater does not exhibit indications of collapse, but those data had low and non-equant resolution, casting doubt on their interpretation. A unit of mostly bright terrain mixed with dark-blue units (in $\mathrm{RGB}=2.0,1.6,1.3 \mu \mathrm{m}$ VIMS color composite images) exhibiting similar infrared color and contrast to Selk crater extends several hundred kilometers east-southeast from the crater. We informally refer to this unit as the Selk "bench" (cf. Fig. 1). Selk crater and the bench are surrounded by the infrared-dark Belet dune fields, which encroach from the southwest (Lorenz and Radebaugh, 2009).

This paper investigates the formation and evolution of the crater and surrounding regions. Cassini VIMS observations used in this investigation are first described, including the use of a radiativetransfer model to reduce these data to generate mosaics or maps of relative albedo. Next, a model used to derive topographic profiles of the rim of Selk crater is presented. The morphology and morphometry along with their implications for the formation and evolution of Selk crater are then discussed. Finally several hypotheses for the genesis of the generally bright terrain of the bench are offered and explored.

\section{Data reduction and analysis}

The Cassini VIMS data presented in this paper were acquired during three Titan flybys: T35 on $08 / 31 / 2007$, T38 on $12 / 05 /$ 2007 , and T40 on 01/05/2008. Five image cubes from T35 were assembled into the regional mosaic shown in Fig. 1. They have resolutions of $9.9-20.5 \mathrm{~km} /$ pixel and incidence and emission angles that range from $\sim 0^{\circ}$ to $50^{\circ}$ and $\sim 0^{\circ}$ to $40^{\circ}$, respectively (Table 1 ). The VIMS mosaic shown in Fig. 2 was made from higher-resolution VIMS data acquired on Titan flybys T38 and T40, with resolution, incidence-angle, and emission-angle ranges of $2.5-12.9 \mathrm{~km} /$ pixel, $\sim 24-44^{\circ}$, and $\sim 3-33^{\circ}$, respectively. Included are the two highestresolution VIMS views of Selk crater (from T40 with a resolution of $2.5-4.4 \mathrm{~km} / \mathrm{pixel}$ ) that are further analyzed and discussed here (e.g., Figs. 3 and 4). The SAR data shown in Fig. 2 were obtained on Titan flybys T36, T39, T41, T44, and T48 (T39 and T48 were high-altitude SAR data referred to as HiSAR). The SAR data that include Selk crater were acquired near the end of the T36 pass and the resolution is quite low (compared to the best SAR images) and highly non-equant $(\sim 3 \times 10 \mathrm{~km} /$ pixel $)$. Additional details regarding the VIMS flybys are available in Barnes et al. (2009).

The VIMS data were first radiometrically calibrated to units of $I /$ $F$ (where $I$ is the observed radiance and $\pi F$ is the incoming solar irradiance) using the procedures described by Brown et al. (2004). Because of the large variations in incidence, emission, and phase angle across and between overlapping cubes (Table 1), prior to mosaicking, the data were further processed to derive model spectral albedo following the method described by Soderblom et al. (2009). Briefly, this model employs the discrete ordinates radiative transfer code, MODTRAN ${ }^{\mathrm{TM}} 5$, developed by Air Force Research Laboratory and Spectral Sciences, Inc. (cf. Berk et al., 2006). This model makes uses of the correlated- $k$ absorption coefficients for $\mathrm{CH}_{4}$ of Irwin et al. (2006), collision-induced absorption of $\mathrm{N}_{2}$ and $\mathrm{H}_{2}$ from McKellar (1989), vertical aerosol haze profile and aerosol shape model presented by Tomasko et al. (2005), and optical constants for laboratory tholins (cf. Sagan and Khare, 1979) reported in Imanaka et al. (2004, 2005). The uniformity across the mosaics in Figs. 1 and 2 demonstrates the success of this procedure, at least in the atmospheric windows at $1.3 \mu \mathrm{m}, 1.6 \mu \mathrm{m}$, and $2.0 \mu \mathrm{m}$ that were used in the color composites shown in those figures.

Inspection of the highest-resolution VIMS views (Figs. 2-4) reveals strong evidence of topographic shading across Selk crater. This is most easily seen in Fig. 4 in the bright material on the east- 


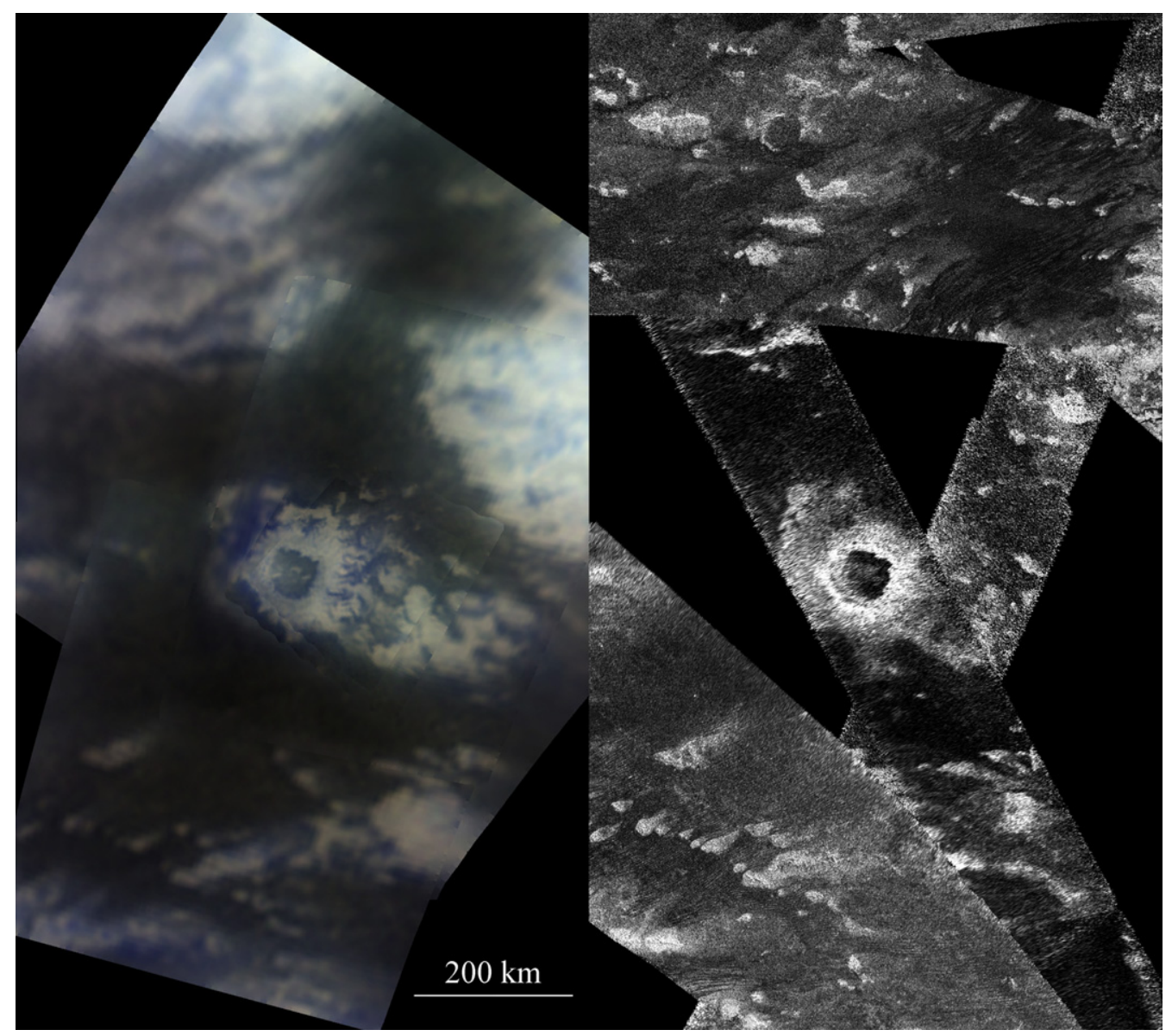

Fig. 2. VIMS and RADAR coverage of Selk crater. Left: VIMS color mosaic (RGB $=2.0,1.6,1.3 \mu \mathrm{m}$ ) of the five cubes acquired during T38 and T40; the highest resolution achieved was during the T40 observation centered on Selk crater, ranging in resolution from 2.5 to $4.4 \mathrm{~km} /$ pixel. Right: mosaic of SAR data acquired during T36, T39, T41, T43, T44, and T48. Selk was observed during the T36 flyby; those data were acquired near the end of the pass and are non-equant and low resolution $(\sim 3 \times 10 \mathrm{~km} /$ pixel)

ern and southern parts of the crater rim. Topographic profiles across the rim were derived from the VIMS data collected through the $2-\mu \mathrm{m}$ and $5-\mu \mathrm{m}$ atmospheric windows (from 5 and 11 spectral channels respectively) using a photoclinometric model (cf. Kirk et al., 2003 and references therein) that assumes a Lambertian surface. The two highest-resolution VIMS cubes of Selk crater were map-projected (sinusoidal), co-registered, and averaged. They were taken only minutes apart and have very similar viewing geometries. Solar illumination was from the east (solar azimuth $\sim 110^{\circ}$ ) and the incidence angle range was $30-35^{\circ}$. The accuracy of topography estimated with photoclinometry relies on the assumption that the surface has uniform albedo along the profile

Table 1

Geometry of VIMS spectral images of the Selk crater region used in this paper.

\begin{tabular}{llclrr}
\hline VIMS cube ID & $\begin{array}{l}\text { Titan } \\
\text { flyby }\end{array}$ & $\begin{array}{l}\text { Resolution } \\
(\mathrm{km})\end{array}$ & $\begin{array}{l}\text { Phase } \\
\text { angle }\left(^{\circ}\right)\end{array}$ & $\begin{array}{l}\text { Incidence } \\
\text { angle }\left(^{\circ}\right)\end{array}$ & $\begin{array}{l}\text { Emission } \\
\text { angle }\left(^{\circ}\right)\end{array}$ \\
\hline CM_1567239055 & T35 & $9.9-10.5$ & $29.7-32.0$ & $0.1-14.1$ & $18.7-39.9$ \\
CM_1567240241 & T35 & $12.9-13.8$ & $25.6-27.5$ & $23.3-47.9$ & $0.1-27.7$ \\
CM_1567241480 & T35 & $16.4-16.8$ & $27.0-28.9$ & $0.1-30.2$ & $4.5-39.4$ \\
CM_1567241662 & T35 & $16.9-17.5$ & $26.1-28.9$ & $0.3-37.9$ & $0.2-39.1$ \\
CM_1567242818 & T35 & $19.9-20.5$ & $25.3-27.8$ & $10.2-49.8$ & $0.2-24.5$ \\
CM_1575509158 & T38 & $6.4-7.4$ & $40.6-43.0$ & $25.2-37.7$ & $9.6-29.8$ \\
CM_1575509654 & T38 & $7.7-8.7$ & $40.8-43.0$ & $25.4-38.6$ & $3.3-21.6$ \\
CM_1578263152 & T40 & $2.5-3.4$ & $31.7-40.3$ & $30.0-35.1$ & $9.2-16.9$ \\
CM_1578263500 & T40 & $3.4-4.4$ & $38.4-44.5$ & $29.2-35.5$ & $7.4-19.6$ \\
CM_1578266417 & T40 & $11.4-12.9$ & $52.1-54.5$ & $23.8-44.2$ & $9.2-33.0$ \\
\hline
\end{tabular}

being integrated; thus, profiles were selected within the bright unit in locations that appear spectrally uniform (cf. Figs. 3 and 4).

Scattering in Titan's atmosphere, which dilutes topographic contrast, further complicates the problem. To account for this, the atmospheric radiative-transfer model described in the previous section was used to estimate the diffuse component of the radiance. The model predicts that the direct component is about $40 \%$ of the radiance at $2 \mu \mathrm{m}$ and $>95 \%$ at $5 \mu \mathrm{m}$ for a $10-20 \%$ reflective surface; the diffuse component along the $I / F$ profile was approximated as $40 \%$ and $0 \%$ of the average $I / F$ for the $2-\mu \mathrm{m}$ and $5-\mu \mathrm{m}$ channels, respectively. The modeled diffuse component was then subtracted from the observed $I / F$ along the profile, yielding the direct component of radiance (unscattered on Sun-surface-S/C path) along the profile (the average $I / F$ (approximating a flat surface) was used because the dark and bright slopes are diffusely lit about equally). The direct component of radiance along the profile can then be used to solve for the slope as follows:

$I / F_{\text {direct }}(x, \lambda)=C * \tilde{\mathbf{n}} \cdot \hat{\mathbf{S}}$

and

$I / F_{\text {direct_flat }}=C * \tilde{\mathbf{n}} \cdot \mathbf{k}$

where $\tilde{\mathbf{n}}$ is the surface normal, $\hat{\mathbf{S}}$ is the vector to the Sun, $\mathbf{k}$ is the local vertical unit vector, and $C$ is a constant that includes the surface albedo and the atmospheric attenuation of the signal (the details are not important as this factor is normalized out). Let $\alpha, \theta$, and $\Phi$ be the angles that define the slope and solar incidence and azimuth and $R(x, \lambda)=I / F_{\text {direct }}(x, \lambda) \div I / F_{\text {direct_flat }}(\lambda)$. Substituting the solutions 

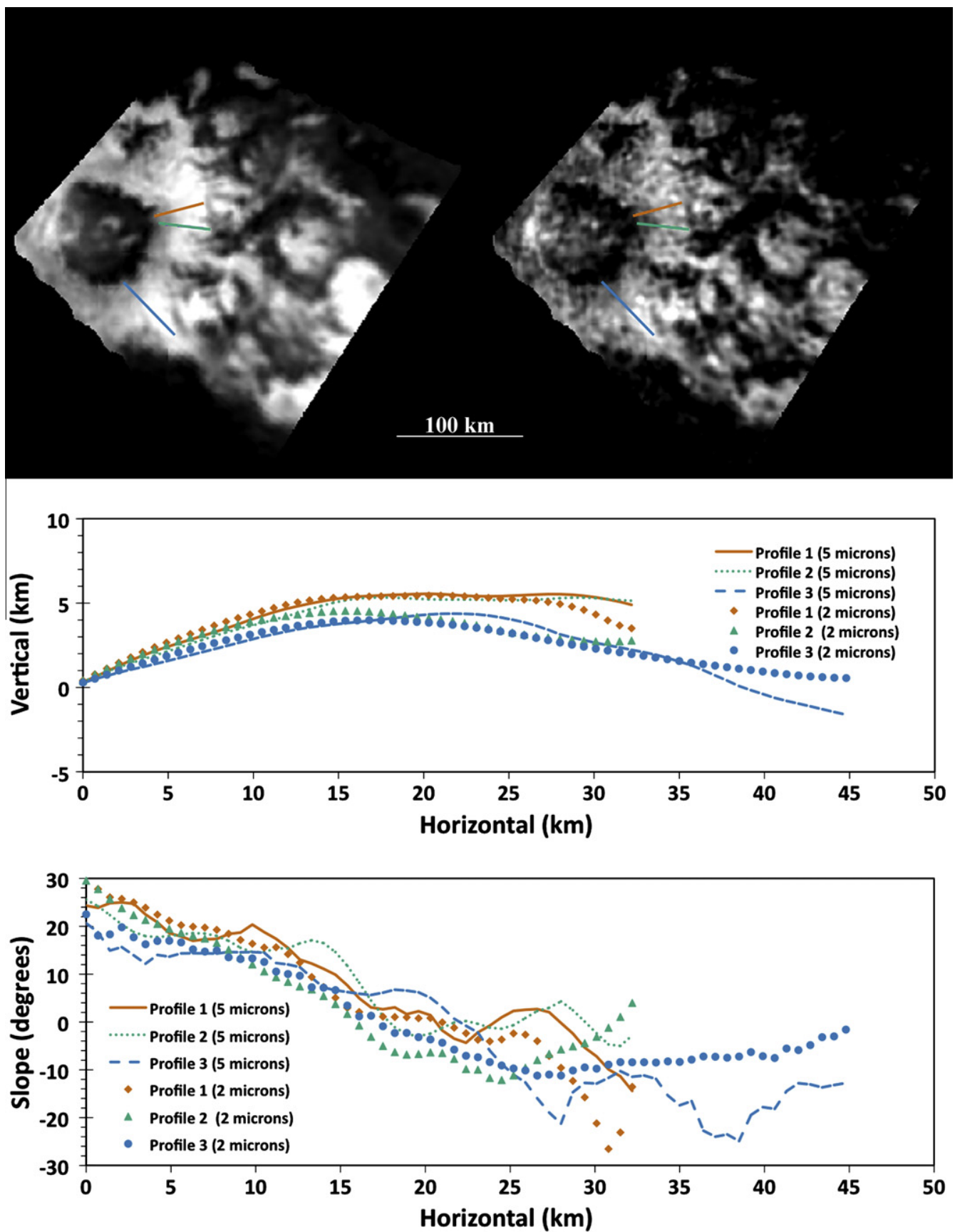

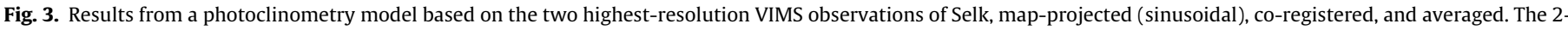

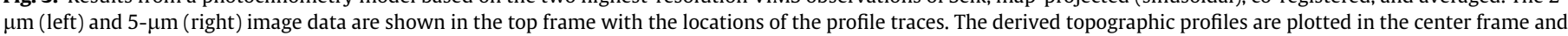
the associated slopes are plotted in the bottom frame. It should be noted that absolute heights are poorly constrained by this photoclinometric model.

for $I / F_{\text {direct }}(x, \lambda)$ and $I / F_{\text {direct_flat }}$ into this equation, $R(x)=\{\sin (\alpha) \sin (\theta)$ $\cos (\Phi)+\cos (\alpha) \cos (\theta)\} / \cos (\theta)$. Solving for $\alpha$ gives,

$\alpha=\operatorname{acos}\left[\left\{R+\Gamma\left(1-R^{2}+\Gamma^{2}\right)^{1 / 2}\right\} /\left(1+\Gamma^{2}\right)\right]$,

where $\Gamma=\tan (\theta) \cos (\Phi) ; \alpha>0$ if $R<1$ and $\alpha<0$ if $R>1$.

Fig. 3 shows topographic elevation and slope profiles across three sections of the crater rim and outer flank derived using this model for data from the atmospheric windows centered at $2 \mu \mathrm{m}$ and $5 \mu \mathrm{m}$. The free variable that was adjusted to level each profile is the $I / F(\lambda)$ of a flat surface; a single value was used to level all profiles together. Hence the precise datum or level for the profiles is not well constrained. While the $5-\mu \mathrm{m}$ data are noisier than those at $2 \mu \mathrm{m}$, atmospheric scattering is significantly reduced at $5 \mu \mathrm{m}$, making the atmospheric corrections more reliable at that wavelength.

\section{Discussion of geomorphology and geology}

\subsection{Selk crater}

Figs. 1 and 2 show regional and higher-resolution, color-composite mosaics of VIMS images of Selk crater; Fig. 2 also shows a mosaic of SAR images of the Selk region. Selk crater appears similar in morphology to Afekan, Ksa, and other unnamed impact craters identified in SAR (Elachi et al., 2006; Wood et al., 2010) and VIMS 

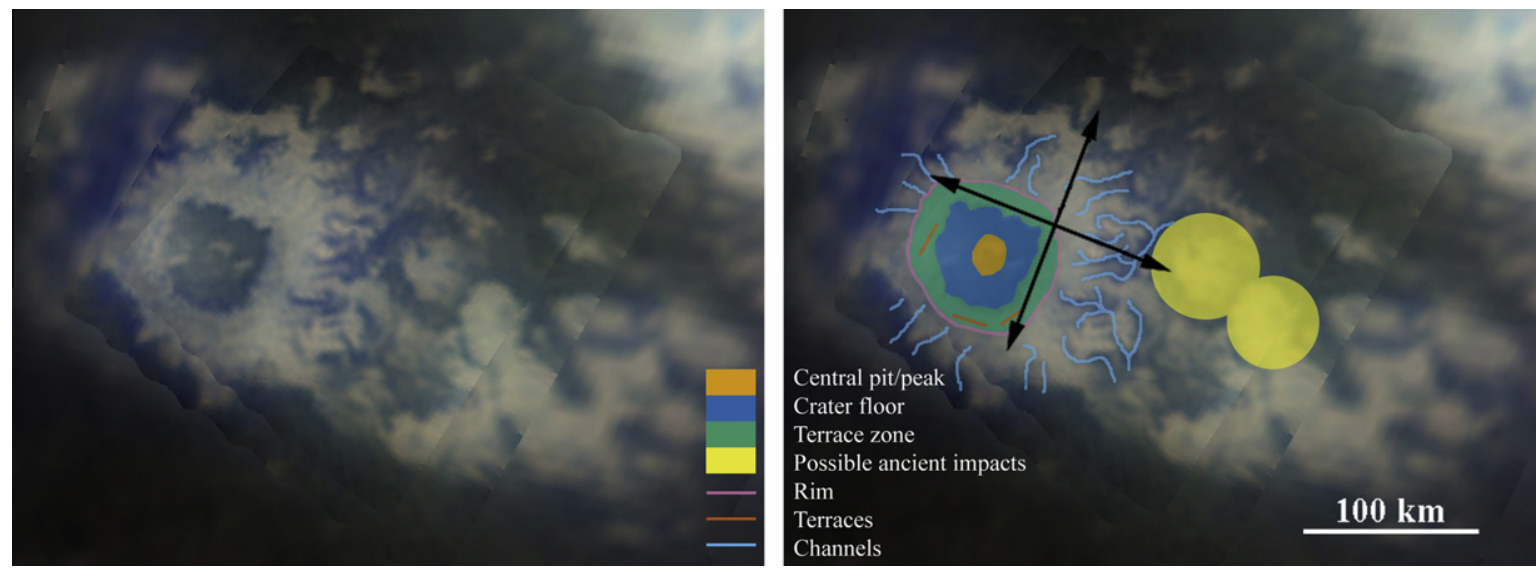

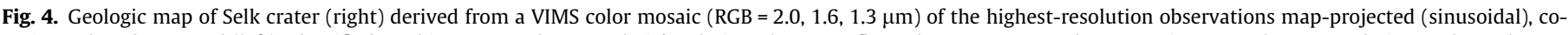

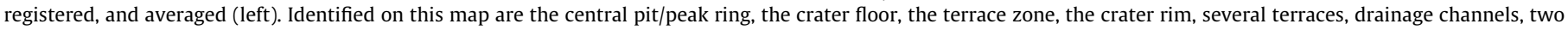
tentatively identified severely degraded impact craters, and the inferred planes of weakness (arrows).

(Soderblom et al., 2007b; Le Mouélic et al., 2008) observations. A geologic sketch map of Selk crater is shown in Fig. 4 and discussed presently. The average image made from the highest-resolution VIMS cubes shown in Figs. 3 and 4 allows for an accurate characterization of the morphometry of this crater.

The rim-crest, as identified in the photoclinometry profiles, is $\sim 90 \mathrm{~km}$ in diameter (Fig. 4). Assuming the sharp albedo boundary apparent in the VIMS and SAR data corresponds to the edge of the crater floor, the crater floor is $\sim 60 \mathrm{~km}$ in diameter (Fig. 4 ). The terrace zone averages $10-15 \mathrm{~km}$ wide horizontally and its slope is nearly double that of the outer flanks of the crater rim (Fig. 3). Terraces on the western and southern walls are visible within the terrace zone (Fig. 4). The terrace zone inferred from the VIMS data corresponds to a very bright halo just exterior to the crater floor in the SAR data (cf. Fig. 2), consistent with this region being exceptionally rough. A central pit or peak, $\sim 20-30 \mathrm{~km}$ in diameter, is also seen in the VIMS data (Fig. 4).

Among the icy satellites of the outer planets, Ganymede and Callisto offer the most appropriate analog to Titan in terms of surface gravity (Ganymede, $143 \mathrm{~cm} \mathrm{~s}^{-2}$; Callisto, $124 \mathrm{~cm} \mathrm{~s}^{-2}$; Titan, $135 \mathrm{~cm} \mathrm{~s}^{-2}$ ) and composition. Crater morphology has been extensively studied for both of these satellites using both Voyager and Galileo data (e.g., Passey and Shoemaker, 1982; Croft, 1983; Schenk, 1991, 1993, 2002; Schenk et al., 2004). Complex impact craters on these satellites are observed to transition from central peaks to central pits at a crater diameter of $\sim 35 \mathrm{~km}$ and from central pits to pits with domes at a diameter of $\sim 60 \mathrm{~km}$ (e.g., Passey and Shoemaker, 1982; Croft, 1983; Schenk, 1993, 2002). If these bodies serve as appropriate analogs, Selk crater should have a central pit and dome. Unfortunately, the VIMS observations of Selk crater are not sufficiently high enough in resolution to differentiate between a pit and peak ring, nor to confirm or deny the presence of a dome.

The ratio of the central-pit/peak-ring diameter to the rim-crest diameter is $\sim 0.2-0.3$, within the range of observed dome-pitto-crater diameter ratios (though on the low end of the observed range) for similarly sized craters on Ganymede and Callisto (cf. Schenk, 1993, Fig. 6a); this is consistent with Selk being a dome crater. The presence of a well-defined rim suggests that Selk crater is not an "anomalous dome" crater (Schenk, 2002; also referred to as Type II penepalimpsests by Passey and Shoemaker (1982) and large dome craters, Schenk (1993)). The crater diameter at which the transition from dome craters to anomalous dome craters on Ganymede has changed from $\sim 60 \mathrm{~km}$ early in the satellite's history (following the emplacement of the bright terrain) to $\sim 150 \mathrm{~km}$ present day, and is interpreted as a reflection of the change in the thermal profile of Ganymede over time (Passey and Shoemaker, 1982; Schenk, 2002). Because Selk, a 90-km crater, is clearly not an anomalous dome crater, it follows that it must have formed when Titan's thermal structure was cooler than Ganymede's in the period following the emplacement of the Ganymede bright terrain.

The terrace zone observed at Selk crater is slightly wider than the widest terrace zones observed on Ganymede and Callisto (cf. Schenk, 1993, Fig. 4), suggesting rim slumping is more significant on Titan than on Ganymede and Callisto. This is likely due to differences in lithospheric properties of these bodies (i.e., composition or, more likely, thermal structure; cf. Schenk, 1991, 1993, 2002). It is worth noting, however, that post-crater-formation eolian and fluvial erosion may contribute to the apparent width of the terrace zone.

The rim of Selk crater appears polygonal; elements in the terrace zone are parallel to this form (cf. Fig. 4). When collapse occurs during complex crater formation, any preexisting planes of weakness, such as joints or faults in the target material, will result in straight segments on the crater walls (Melosh, 1989). We submit that the straight segments observed at Selk crater, oriented at azimuths of $21^{\circ}$ and $122^{\circ}$ east of north, are parallel planes of weakness that existed in the crust prior to the formation of Selk crater and have resulted in the observed polygonal appearance of the crater.

The interior of Selk crater appears further modified by erosion (cf. Fig. 4). The inner rim of the crater exhibits chutes in the northern and eastern walls of the crater, giving it a scalloped appearance; the orientation of the scallops is consistent with erosion from a southwesterly wind. Lorenz and Radebaugh (2009) report southwesterly winds for regions to the southwest and north of Selk crater. Unfortunately, there is not yet highresolution Cassini SAR coverage in the immediate vicinity of Selk crater from which dune orientations, and therefore winds more local to Selk can be inferred.

The outer rim appears dissected by fluvial erosion. The highestresolution VIMS cubes reveal what appear to be numerous short drainage valleys with at least 2 nd and 3 rd order dendritic drainage networks on the eastern flank of the crater where the bench contacts the crater (cf. Fig. 4). The morphology of these drainage networks is consistent with fluvial erosion resulting from methane rain hypothesized to cause drainage networks observed elsewhere on Titan (e.g., Tomasko et al., 2005; Soderblom et al., 2007a; Barnes et al., 2007b; Jaumann et al., 2008; Lorenz et al., 2008). In contrast, the valleys observed on the north, south and western flanks of the 
crater are rather straight with no higher-order branches observed, consistent with fluvial erosion of relatively steep slopes (Phillips and Schumm, 1987). In false-color composites of Selk crater shown in Figs. 1 and 2 ( $R G B=2.0,1.6,1.3 \mu \mathrm{m}$; constructed from five, four, and two spectral channels respectively), the channels appear dark blue relative to the bright terrain and terminate in dark-blue patches. The infrared spectral signature of these blue units is consistent with enrichment in water ice and they appear to be deltas made of sediment transported by liquid methane (cf. Soderblom et al., 2007b) deposited at the mouths of these channels, consistent with the conclusions of Barnes et al. (2007b), Jaumann et al. (2008), and Soderblom et al. (2009).

\subsection{The "bench"}

A unit of mostly bright terrain, to which we informally refer to as the "bench," that exhibits similar infrared-color variation and contrast to Selk crater, extends east-southeast from the crater several hundred kilometers (cf. Fig. 1). The proximity and similarity in size and infrared color between the bench and Selk crater suggests the origins of the two features are correlated.

One possibility is that Selk crater acts as a topographic obstacle within the Belet dune field that creates a wind shadow, keeping a region of the surface leeward of Selk crater free from dunes; such features have been reported in SAR observations (Radebaugh et al., 2008, 2009; Fig. 2). In the false-color composite (with $\mathrm{RGB}=2.0,1.6,1.3 \mu \mathrm{m}$ ) shown in Fig. 2, the entire region surrounding Selk crater and the bench is dark brown, a spectral character that has been shown to strongly correlate with the dunes observed in SAR images (Soderblom et al., 2007b; Barnes et al., 2007a). Rather than being co-aligned with the long axis of the bench, however, the mean-peak wind direction inferred by Lorenz and Radebaugh (2009) for two regions located $\sim 500 \mathrm{~km}$ southwest and $\sim 500 \mathrm{~km}$ north of Selk crater (cf. Fig. 2 ) is offset by $\sim 45^{\circ}$ from this axis. For the bench to simply be a dune free area in the wind shadow of Selk, winds local to Selk crater need to be locally offset by $\sim 45^{\circ}$ from the observed regional average winds.

If the wind directions reported by Lorenz and Radebaugh (2009) for the regions southwest and north of Selk crater represent the current average regional winds, and the dunes are approaching this feature from the southwest, the southern margin of the bench must have significant relief to stand off the dunes. If this interpretation is correct, we should see the dunes approach the bench from the southwest and divert to the east, paralleling the bench along its southern margin near its eastern extent. In the VIMS data, the transition along the southern margin of this structure from the darkbrown (i.e., dunes) unit to the bright unit is $\sim 40 \mathrm{~km}$ wide. Using the relationships derived by Lorenz and Radebaugh (2009), who examined the slopes of obstacles in Titan dune fields using SAR images, the southern margin of the bench must have a height of at least $\sim 200 \mathrm{~m}$ in order to divert the dunes. Similar relief is observed in SAR stereogrammetry of other radar-bright dune-free features in the region (Kirk et al., 2009).

Because bright features in the bench have some morphologic similarities to flow features, a possible interpretation is that this feature is the result of impact-induced cryovolcanism. In that model, underlying material is melted through decompression immediately following an impact event. The resultant magma then flows up impact-induced fractures in the surface and erupts onto the surface. The work of Ivanov and Melosh (2003), however, demonstrates that such processes could only occur if a large impact were to strike a preexisting hotspot, an event they conclude is highly improbable.

A more likely process that could produce flow-like features associated with an impact is a thermally fluidized-ejecta flow, similar to the process suggested for the emplacement of the "bright crater outflows" observed on Venus (e.g., Phillips et al., 1991; Schaber et al., 1992). High surface gravity and impact velocities at Venus, and to a lesser extent, high surface temperatures and pressures, result in greater-than-average impact-melt fractions associated with venusian craters (Vickery and Melosh, 1991). The increased melt fraction and the high surface and atmospheric temperatures result in ejecta outflows associated with many venusian craters; these outflows are extended, low-viscosity flows (in one case extending nearly seven crater diameters from the crater rim), with no detectable relief at their margins (Phillips et al., 1991; Schaber et al., 1992). While some research has suggested that impacts into icy satellites might produce substantial impact melt (e.g., Pierazzo et al., 1997), Lorenz (1997) predicts that, because the surface and atmospheric temperatures on Titan relative to the melting point of its water ice crust are relatively lower than equivalent temperatures on Venus, ejecta blankets will be more likely than long, venusian-like ejecta flows (e.g., Sinlap crater; Elachi et al., 2006). The bright, flow-like features across the bench having relief sufficient to standoff the encroachment of the dunes would not be consistent with the low viscosities implied for venusian ejecta flows, but rather with flows of relatively higher viscosity.

Another characteristic of the bright and dark materials on the bench east of Selk is the presence of circular features similar in appearance to remnants of ancient impact craters (cf. Fig. 4). This could be a cluster of craters that resulted from atmospheric disruption of an impacting object (e.g., Venus; cf. Schaber et al., 1992), but the probability of a crater field of such size (each crater being on order $50-100 \mathrm{~km}$ in diameter) is quite low; Korycansky and Zahnle (2005) predict $<0.1$ such events should occur per Ga. Additionally, the relatively pristine appearance of the Selk crater as compared to the other tentatively identified craters suggests that if these are impact craters, they are much older than Selk and could be remnant of Titan's early cratered highlands (as occur on most of the icy satellites of the outer planets). This result does not preclude the possibility that the bench represents a fluidized-ejecta flow; if the flow is only moderately viscous, it will not necessarily mask the underlying topography.

Turning attention to the large-scale structure of the bench, a generally streamlined shape is apparent (cf. Fig. 1). It is possible that Selk crater and the bench represent a streamlined upland. Streamlined forms are common on Earth and Mars, and are interpreted to be erosive features associated with floods from large outflow channels (e.g., Masursky et al., 1977; Baker, 1978). They are thought to form when a fluid flows around an obstacle, resulting in the erosion of material downstream of the obstacle. It should be noted, however, that this feature is several times larger than the largest martian streamlined islands.

Baker (1979) suggested and Komar (1983) confirmed experimentally, that the morphology of streamlined islands approximate a minimum-drag form, with a length-to-width ratio $(L / W)$ of $\sim 3-4$ for landforms shaped by liquid water; the observed $L / W$ for Selk crater and the bench is only about $\sim 2.5$. In studies of floods forming elongated streamlined islands observed on Mars, Baker and Kochel (1978) demonstrated empirically that $L / W$ is directly dependent on the Reynolds number (Re), which depends directly on the density and velocity and inversely on the viscosity of the flowing fluid. Methane rain on Titan's surface will be $\sim 1 / 2$ as dense and $\sim 1 / 5$ as viscous as water at STP (Lorenz et al., 2003) resulting in a larger Re than liquid water at STP for a given velocity. The lower surface gravity on Titan, however, will result in slower flow velocities for a given slope (e.g., Burr et al., 2006), decreasing Re; the net result is that the characteristic $L / W$ for streamlined forms on Titan generated by flowing methane will be similar to terrestrial and martian values: $3-4$. If Selk crater and the bench formed as a streamlined island, they likely formed in the presence of a more 
viscous fluid than pure methane. Liquids released from lakes on Ti$\tan$ (e.g., via a dam breach) will likely be composed of a mixture of methane, ethane, and nitrogen (Lunine et al., 1983) and could have much higher viscosities than pure methane; dissolved higherorder hydrocarbons or suspended particulates would act to increase viscosity further (cf. Lorenz et al., 2009); lower surface temperatures, as might be experienced in an earlier geologic epoch, would also result in higher viscosities (Lorenz et al., 2010).

The possibility that this landform is the result of large-scale flooding from west-northwest to east-southeast is further supported by the work of Soderblom et al. (2007a), who suggest, from Huygens Probe Descent Imager/Spectral Radiometer images, that large-scale flooding from west-to-east has occurred across the plains at the Huygens landing site, located $\sim 800 \mathrm{~km}$ south-southeast of Selk.

\section{Summary and conclusions}

We conclude from our investigation of VIMS data that Selk crater, a bright-rimmed crater observed on Titan, is a fairly typical complex impact crater on an icy satellite. The rim crest of Selk crater measures $\sim 90 \mathrm{~km}$ in diameter. Assuming the sharp albedo boundary seen in the VIMS images defines the edge of the crater floor, the floor is $\sim 60 \mathrm{~km}$ in diameter, and the terrace zone averages $\sim 10-15 \mathrm{~km}$ in width. A central pit/peak ring, approximately $\sim 20-30 \mathrm{~km}$ in diameter, is evident in the VIMS observations.

The terrace zone is slightly wider than those observed on Ganymede and Callisto (the most similar icy satellites in terms of surface gravity) and may reflect differences in lithospheric thermal structure and/or composition. Craters of this size on Ganymede and Callisto exhibit central domes within the central pits. While the VIMS data are not of sufficient resolution to identify such a feature, the ratio of the central-pit/peak-ring diameter to the rimcrest diameter is consistent with craters of this size on Ganymede and Callisto (cf. Schenk, 1993, Fig. 6a), all of which are dome craters (Passey and Shoemaker, 1982). Selk crater does not exhibit any of the morphologic signatures of an anomalous dome crater (Passey and Shoemaker, 1982) and therefore must have formed when the thermal structure of Titan was cooler than that of Ganymede early in that satellite's history (in the period following the emplacement of the bright terrain on Ganymede).

The polygonal morphology of Selk crater is likely the result of two nearly perpendicular planes of weakness at azimuths of $21^{\circ}$ and $112^{\circ}$ east of north that existed in the crust prior to the formation of Selk crater and controlled collapse of the crater rim. The terrace zone identified in VIMS data appears very bright in SAR data, consistent with the surface of the terrace zone being extremely rough. The slope of the terrace zone is nearly double that of the outer flanks of the crater rim. Terraces on the western and southern walls are visible within this region. That Wood et al. (2009) report that Selk crater lacks evidence of collapse does not contradict our results because they based their conclusions on SAR images acquired late in the T36 pass that are of lower resolution and quality than SAR data acquired much closer to Titan.

The inner rim of Selk crater appears scalloped, with chutes cut into the northern and eastern parts of the rim. This likely evidences eolian erosion, resulting from a wind similar in direction to the winds predicted by Lorenz and Radebaugh (2009) for regions several hundred kilometers southwest and north of Selk crater. The eastern flank of Selk crater appears dissected by numerous dendritic drainage networks, probably resulting from fluvial erosion by methane rain. The channels appear dark blue relative to the bright unit in VIMS color composites (RGB = 2.0, 1.6, $1.3 \mu \mathrm{m}$ ) and the channel terminations are frequently associated with dark-blue regions, the infrared spectral signature of which has been suggested to be consistent with enrichment in water ice (e.g., Soderblom et al., 2007b). These units are interpreted to be deltas of water-ice-rich sediment transported by liquid methane and deposited at the mouths of these channels. Barnes et al. (2007b), Jaumann et al. (2008), and Soderblom et al. (2009) drew similar conclusions analyzing VIMS observations of other regions of Titan.

A generally optically bright landform (the "bench"), similar in width and infrared-color variation and contrast to Selk crater, extends $\sim 200 \mathrm{~km}$ east from Selk crater. The large Belet dune field surrounds both Selk and the bench; dunes are resolved in SAR images acquired few hundred kilometers to the north and southwest, while the region locally surrounding Selk crater and the bench is dark brown in false-color composites ( $\mathrm{RGB}=2.0,1.6,1.3 \mu \mathrm{m})$ and is therefore inferred to be dunes (cf. Soderblom et al., 2007b; Barnes et al., 2007a). The bench must have sufficient relief along its southern margin to obstruct dunes encroaching from the southwest; a minimum height of $\sim 200 \mathrm{~m}$ is estimated for this feature using the relationships reported by Lorenz and Radebaugh (2009). Two possible ancient impact craters are identified on the bench; if so, they evidence a remnant of ancient cratered crust. Two working hypotheses for the origin of the elongated bench seem reasonable: a fluidized-ejecta flow (a class intermediate to ejecta blankets and bright crater outflows observed on Venus; cf. Phillips et al., 1991; Schaber et al., 1992) or a streamlined upland that formed as result of the flow of an erosive fluid from west to east, similar to the martian streamlined islands associated with large outflow channels, formed during catastrophic flooding (cf. Baker, 1978, 1979) and subsequently dissected by fluvial erosion. The erosive fluid must be higher in viscosity than pure methane at current Titan temperatures. Neither of these precludes the possibility that the bench is a remnant of ancient cratered highland.

\section{Acknowledgments}

We express our gratitude to the entire Cassini Project with specific thanks to the VIMS Operations Group for years of effort in returning these data. We also thank Ralph Lorenz for his helpful discussion regarding fluid flows on Titan. We are grateful to Alex Hayes and Jani Radebaugh for their thoughtful reviews of this manuscript. This work was supported by the Cassini Project, managed by the Jet Propulsion Laboratory, California Institute of Technology under contract with NASA.

\section{References}

Baker, V.R., 1978. The Spokane flood controversy and the martian outflow channels. Science 202, 1249-1256.

Baker, V.R., 1979. Erosional processes in channelized water flows on Mars. J. Geophys. Res. 84 (B14), 7985-7993.

Baker, V.R., Kochel, R.C., 1978. Morphometry of streamlined forms in terrestrial and martian channels. Proc. Lunar. Sci. Conf. IX, 3193-3203.

Barnes, J.W., Brown, R.H., Soderblom, L., Buratti, B.J., Sotin, C., Rodriguez, S., Le Mouèlic, S., Baines, K.H., Clark, R., Nicholson, P., 2007a. Global-scale surface spectral variations on Titan seen from Cassini/VIMS. Icarus 186, 242-258. doi:10.1016/j.icarus.2006.08.021.

Barnes, J.W., and 19 colleagues, 2007b. Near-infrared spectral mapping of Titan's mountains and channels. J. Geophys. Res. 112, E11006. doi:10.1029/ 2007JE002932.

Barnes, J.W., and 31 colleagues, 2009. VIMS spectral mapping observations of Titan during the Cassini prime mission. Planet. Space Sci. 57, 1950-1962. doi:10.1016/j.pss.2009.04.013.

Berk, A., and 15 colleagues, 2006. MODTRAN ${ }^{\mathrm{TM}}$ 5: 2006 Update. In: Shen, S.S., Lewis, P.E. (Eds.), Algorithms and Technologies for Multispectral, Hyperspectral, and Ultraspectral Imagery XII, Proceedings of the SPIE 6233, 62331F. doi:10.1117| 12.665077.

Brown, R.H., and 21 colleagues, 2004. The Cassini Visual and Infrared Mapping Spectrometer (VIMS) investigation. Space Sci. Rev. 115, 111-168. doi:10.1007/ s11214-004-1453-X.

Burr, D.M., Emery, J.P., Lorenz, R.D., Collins, G.C., Carling, D.A., 2006. Sediment transport by liquid surficial flow: Application to Titan. Icarus 180, 235-242. doi:10.1016/j.icarus.2005.11.012. 
Croft, S.K., 1983. A proposed origin for palimpsests and anomalous pit craters on Ganymede and Callisto. J. Geophys. Res. 88, B71-B89.

Elachi, C., and 21 colleagues, 2004. RADAR, The Cassini Titan RADAR mapper. Space Sci. Rev. 115, 71-110. doi:10.1007/s11214-004-1438-9.

Elachi, C., and 34 colleagues, 2006. Titan Radar Mapper observations from Cassini's T3 fly-by. Nature 441, 709-713. doi:10.1038/nature04786.

Imanaka, H., Khare, B.N., Elsila, J.E., Bakes, E.L.O., McKay, C.P., Cruikshank, D.P., Sugita, S., Matsui, T., Zare, R.N., 2004. Laboratory experiments of Titan tholin formed in cold plasma at various pressures: Implications for nitrogencontaining polycyclic aromatic compounds in Titan haze. Icarus 168, 344366. doi:10.1016/j.Icarus.2003.12.014.

Imanaka, H., Khare, B.N., McKay, C.P., Cruikshank, D.P., 2005. Complex refractive indices of tholins produced from various initial gas mixtures and formation pressures: Implications for Titan, the early Earth, and the outer Solar System bodies. Bull. Am. Astron. Soc. 37, 772 (abstract).

Irwin, P.G.J., Sromovsky, L.A., Strong, E.K., Sihra, K., Teanby, N.A., Bowles, N., Calcutt, S.B., Remedios, J.J., 2006. Improved near-infrared methane band models and $k$ distribution parameters from 2000 to $9500 \mathrm{~cm}^{-1}$ and implications for interpretation of outer planet spectra. Icarus 181, 309-319. doi:10.1016/ j.Icarus.2005.11.003.

Ivanov, B.A., Melosh, H.J., 2003. Impacts do not initiate volcanic eruptions: Eruptions close to the crater. Geology 31, 869-872. doi:10.1130/G19669.1.

Jaumann, R., Neukum, G., 2009. The surface age of Titan. Lunar. Planet. Sci. XL, 1641 (abstract).

Jaumann, R., and 19 colleagues, 2008. Fluvial erosion and post-erosial processes on Titan. Icarus 197, 526-538. doi:10.1016/j.Icarus.2008.06.002.

Kirk, R.L., Barrett, J.M., Soderblom, L.A., 2003. Photoclinometry Made Simple? ISPRS Working Group IV/9, "Advances in Planetary Mapping 2003", Houston.

Kirk, R.L., 16 colleagues, and the Cassini RADAR Team, 2009. Three-dimensional Views of Titan's Diverse Surface Features from Cassini RADAR Stereogrammetry. Lunar. Planet. Sci. XL, 1414 (abstract).

Komar, P.D., 1983. Shapes of streamlined islands on Earth and Mars: Experiments and analyses of the minimum-drag form. Geology 11, 651-654. doi:10.1130/ 0091-7613(1983)11.

Korycansky, D.G., Zahnle, K.J., 2005. Modeling crater populations on Venus and Titan. Planet. Space Sci. 53, 695-710. doi:10.1016/j.Pss.2005.03.002.

Le Mouélic, S., and 17 colleagues, 2008. Mapping and interpretation of Sinlap crater on Titan using Cassini VIMS and RADAR data. J. Geophys. Res. 113, E04003. doi:10.1029/JE002965

Lorenz, R.D., 1997. Impacts and cratering on Titan: A pre-Cassini view. Planet. Space Sci. 45, 1009-1019. doi:10.1016/S0032-0633(97)00085-8.

Lorenz, R.D., Radebaugh, J. 2009. Global pattern of Titan's dunes: Radar survey from the Cassini prime mission. Geophys. Res. Lett. 36, L03202. doi:10.1029/ 2008GL036850.

Lorenz, R.D., Kraal, E., Asphaug, E., Thomson, R.E., 2003. The Seas of Titan. Eos Trans. AGU, 84 (14). doi:10.1029/2003EO140002.

Lorenz, R.D., and 11 colleagues, 2007. Titan's young surface: Initial impact crater survey by Cassini RADAR and model comparison. Geophys. Res. Lett. 34, L07204. doi:10.1029/2006GL028971.

Lorenz, R. D., 14 colleagues, and the Cassini RADAR Team, 2008. Fluvial channels on Titan: Initial Cassini RADAR observations. Planet. Space Sci. 56, 1132-1144. doi:10.1016/j.pss.2008.02.009.

Lorenz, R.D., Newman, C., Lunine, J.I., 2010. Threshold of wave generation on Titan's lakes and seas: Effect of viscosity and implications for Cassini observations. Icarus 207, 932-937.

Lunine, J.I., Stevenson, D.J., Yung, Y.L., 1983. Ethane ocean on Titan. Science 222, 1229-1230. doi:10.1126/science.222.4629.1229.

Masursky, H., Boyce, J.M., Dial, A.L., Schaber, G.G., Strobell, M.E., 1977. Classification and time of formation of martian channels based on Viking data. J. Geophys. Res. 82, 4016-4038.

McKellar, A.R.W., 1989. The spectrum of gaseous methane at $77 \mathrm{~K}$ in the $1.1-2.6-\mu \mathrm{m}$ region: A benchmark for planetary astronomy. Can. J. Phys. 67, 1027-1035.

Melosh, H.J., 1989. Impact Cratering: A Geologic Process. Oxford Univ. Press, New York, p. 84
Melosh, H.J., Ivanov, B.A., 1999. Impact Crater Collapse. Annu. Rev. Earth Planet. Sci. 27, 385-415. doi:10.1146/annurev.earth.27.1.385.

Passey, R.R., Shoemaker, E.M., 1982. Craters and basins on Ganymede and Callisto: Morphological indicators of crustal evolution. In: Morrison, D. (Ed.), Satellites of Jupiter. The Univ. of Arizona Press, Tucson, pp. 379-434.

Phillips, L.F., Schumm, S.A., 1987. Effect of regional slope on drainage networks. Geology 15, 813-816.

Phillips, R.J., Arvidson, R.E., Boyce, J.M., Campbell, D.B., Guest, J.E., Schaber, G.G., Soderblom, L.A., 1991. Impact craters on Venus - Initial analysis from Magellan. Science 252, 288-297. doi:10.1126/science.252.5003.288.

Pierazzo, E., Vickery, A.M., Melosh, H.J., 1997. A reevaluation of impact melt production. Icarus 127, 408-423. doi:10.1006/icar.1997.5713.

Pike, R.J., 1980. Formation of complex impact craters: Evidence from Mars and other planets. Icarus 43, 1-19. doi:10.1016/0019-1035(80)90083-4.

Porco, C.C., and 19 colleagues, 2004. Cassini imaging science: Instrument characteristics and anticipated scientific investigations at Saturn. Space Sci. Rev. 115, 363-497. doi:10.1007/s11214-004-1456-7.

Porco, C.C., and 35 colleagues, 2005. Imaging of Titan from the Cassini spacecraft. Nature 434, 159-168. doi:10.1038/nature03436.

Radebaugh, J., 14 colleagues, and the Cassini RADAR Team, 2008. Dunes on Titan observed by Cassini Radar. Icarus 194, 690-703. doi:10.1016/ j.Icarus.2007.10.015.

Radebaugh, J., Lorenz, R., Farr, T., Paillou, P., Savage, C., Spencer, C., 2009. Linear dunes on Titan and Earth: Initial remote sensing comparisons. Geomorphology, in press. doi:10.1016/j.Geomorph.2009.02.022.

Sagan, C., Khare, B.N., 1979. Tholins: Organic chemistry of interstellar grains and gas. Nature 277, 102-107. doi:10.1038/277102a0.

Schaber, G.G., Strom, R.G., Moore, H.J., Soderblom, L.A., Kirk, R.L., Chadwick, D.J., Dawson, D.D., Gaddis, L.R., Boyce, J.M., Russell, J., 1992. Geology and distribution of impact craters on Venus - What are they telling us? J. Geophys. Res. 97 (E8), 13257-13301.

Schenk, P.M., 1991. Ganymede and Callisto: Complex crater formation and planetary crusts. J. Geophys. Res. 96 (E1), 15635-15664.

Schenk, P.M., 1993. Central pit and dome craters: Exposing the interiors of Ganymede and Callisto. J. Geophys. Res. 98 (E4), 7475-7498.

Schenk, P.M., 2002. Thickness constraints on the icy shells of the Galilean satellites from a comparison of crater shapes. Nature 417, 419-421. doi:10.1038/417419.

Schenk, P.M., Chapman, C.R., Zahnle, K., Moore, J.M., 2004. Ages and interiors: Cratering record of the Galilean satellites. In: Bagenal, F., Dowling, T., McKinnon, W. (Eds.), Jupiter the Planet, Satellites and Magnetosphere. Cambridge Univ. Press, Cambridge, pp. 427-456.

Soderblom, L.A., and 18 colleagues, 2007a. Topography and geomorphology of the Huygens landing site on Titan. Planet. Space Sci. 55, 2015-2024. doi:10.1016/ j.pss.2007.04.015.

Soderblom, L.A., and 26 colleagues, 2007b. Correlation between Cassini VIMS spectra and RADAR SAR images: Implications for Titans' surface composition and character of the Huygens Probe Landing Site. Planet. Space Sci. 55, 20252036. doi:10.1016/j.Pss.2007.04.014.

Soderblom, L.A., and 12 colleagues, 2009. The Geology of Hotei Regio, Titan Correlation of Cassini VIMS and RADAR. Icarus 204, 610-618. doi:10.1016/ j.icarus.2009.07.033.

Stofan, E. R., and 35 colleagues, 2006. Mapping of Titan: Results from the first Titan radar passes. Icarus 185, 443-456. doi:10.1016/j.Icarus.2006.07.015.

Tomasko, M.G., and 39 colleagues, 2005. Rain, winds and haze during the Huygens probe's descent to Titan's surface. Nature 438, 765-778. doi:10.1038/ nature 04126 .

Vickery, A.M., Melosh, H.J., 1991 Production of impact melt in craters on Venus, Earth, and the Moon. Lunar. Planet. Sci. XXII, 1443-1444 (abstract).

Wood, C.A., Kirk, R., Lorenz, R.D., 2009. Numbers, distributions and morphologies of impact craters on Titan. Lunar. Planet. Sci. XL, 2242 (abstract).

Wood, C.A., Lorenz, R., Kirk, R., Lopes, R., Mitchell, K., Stofan, E., 2010. Impact craters on Titan. Icarus 206, 334-344. doi:10.1016/j.icarus.2009.08.021. 